\title{
3 Dimensional Microscopy, Large Volume Serial Block Face Imaging in the SEM
}

\author{
Mancuso, Joel ${ }^{1}$ \\ ${ }^{1}$ Gatan Inc, Pleasanton, CA jmancuso@gatan.com
}

An essential biological question is "how is the three-dimensional geometry of cellular structures organized?" Life science researchers have two options for pursuing this question at the transmission electron microscope (TEM) level, tomography, and serial section imaging. Although tomography has excellent resolution, the field of view is limited and it is impractical for large volume acquisitions. Serial sectioning is used for obtaining large volume threedimensional 3D electron microscopic data, but the technique is a highly skilled time consuming process prone to human error. Serial block-face scanning electron microscopy (SBFSEM) is a new automated technique in which a scanning electron microscope is used to obtain serial images. Bridging the gap between ultrahigh resolution tomography and fluorescence microscopy, SBFSEM provides a streamlined and automated 3D data acquisition process. A microtome equipped with a diamond knife is mounted inside the chamber of the SEM and shaves the surface of the sample in between imaging. Images are collected using a back scatter (BS) electron detector which provides a classic TEM-like image. The SBFSEM imaging process is completely automated allowing for large volume acquisition in a timely manner without the risk of losing sections.

In conjunction with 64bit DigitalMicrograph, SBFSEM is no longer memory limited, and can collect data sets unprecedented by any other 3D imaging technique. Using stage montage $3 \mathrm{View}$ can acquire images from the entire block face, up to $1 \mathrm{~mm}^{2}$, with a pixel size of less than $5 \mathrm{~nm}$. In addition SBFSEM cutting techniques have approached a thinner slice thickness down to $2.5 \mathrm{~nm}$ thick for material samples such as aluminium alloys, and $7 \mathrm{~nm}$ for plastic embedded biological samples.

References:[1] Denk W, Horstmann H. (2004). Serial block-face scanning electron microscopy to reconstruct three-dimensional tissue nanostructure. PLoS Biol. 2/e329:1900- 1909; 329. 

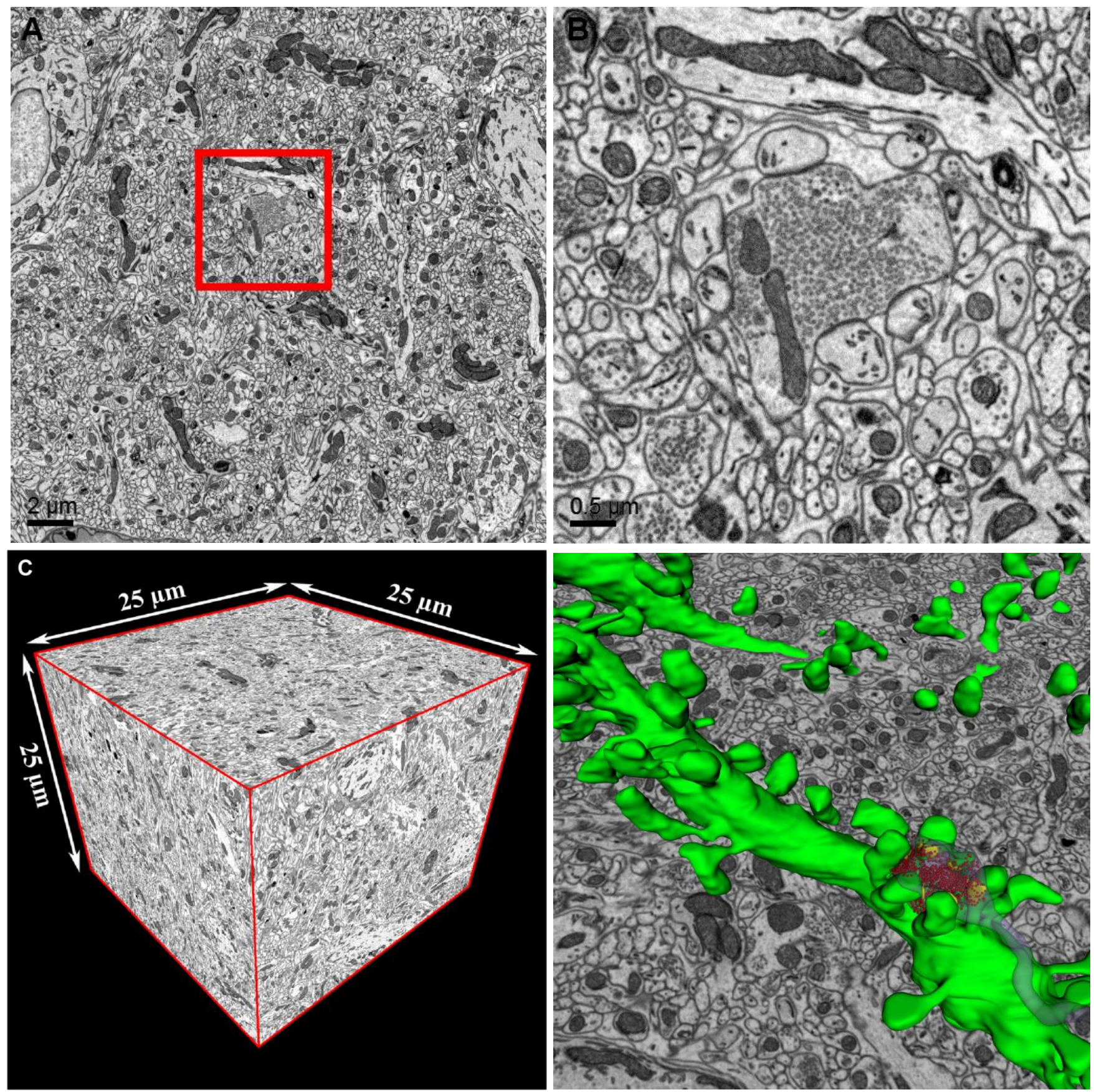

Figure A. A low magnification overview from the volumetric data set in Figure 1. The original data set was $4096 \times 4096$ pixels, and contained 500 serial images.

Figure B. A high magnification image from the region indicated in Figure 2. Note individual synaptic vesicles are resolved.

Figure C. A 15,625 $\mu^{3}$ volumetric data set containing 500 serial images of mouse brain obtained using 3 View $^{\circledR}$ installed on a FEI Quanta ${ }^{\text {TM}} 200$ FEG SEM.

Figure D. Volumetric segmentation of the raw data showing synapses in yellow, a dendrite in green, vesicles in red. 